\title{
Unidad sintética y apercepción
}

\author{
María del Carmen Paredes Martín \\ Universidad de Salamanca \\ paredes@usal.es
}

En la primera edición de la Crítica de la razón pura, Kant expone tres tipos de síntesis, examina la naturaleza de los actos que realizan actividad sintética y las implicaciones que se derivan para la unidad de la conciencia. Asimismo, introduce la noción de «apercepción trascendental» como capacidad de unificar una diversidad de objetos de la representación en una noción de objeto. Todo esto aparece también conectado con la conciencia de sí como sujeto de nuestras representaciones.

Una de las primeras cuestiones que plantea la «Deducción trascendental» de la primera edición es la escasa presencia del término autoconciencia (Selbsbewusstsein), sobre todo en comparación con el de conciencia. Esto abre de inmediato dos posibilidades de interpretación de las que se siguen ciertas consecuencias: una, es la de atenerse literalmente a los términos kantianos; y otra, la de interpretar en determinados contextos que la conciencia incluye también la conciencia de sí o autoconciencia. A este respecto, se puede plantear concretamente si para Kant la conciencia de objeto requiere la conciencia de sí, y viceversa, si la conciencia de sí requiere tener conciencia de algún 
objeto. Porque si entendemos por bewusstsein sólo conciencia, entonces tendremos por lo general una conexión entre la conciencia de objeto y la unidad de la conciencia, pero no con la autoconciencia. Esto vendría a significar que una representación requiere la unidad de la conciencia, pero no necesariamente la autoconciencia.

Sin embargo, es interpretación ampliamente admitida que cuando Kant habla de la conciencia como condición de la experiencia se está refiriendo a la conciencia de sí, si no como autoconciencia actual, al menos como autoconciencia potencial.

Respecto a si la conciencia de sí requiere tener conciencia de algún objeto, cabe decir que esto es posible, hasta cierto punto, en la primera edición de la Crítica, aunque no en la segunda. Estas diferencias que se acaban de señalar, por citar sólo algunas, indican que un estudio detallado de cualquier aspecto de la Crítica de la razón pura requeriría tener en cuenta los planteamientos de las dos versiones y sus relaciones internas, cosa que en este momento no resultaría completamente viable. En todo caso, la importancia de la reelaboración que Kant llevó a cabo tiene especial alcance para el tema de este trabajo, y por ello me voy a atener a la segunda edición, que Kant consideró como la expresión más completa de su pensamiento.

\section{Unidad sintética y deducción}

Afirma Kant en la segunda sección de la «Deducción Trascendental» que «la unidad sintética de la conciencia es por tanto una condición objetiva de todo conocimiento, no de algo que yo mismo necesitara para conocer un objeto, sino algo bajo lo cual tiene que estar toda intuición para convertirse en objeto para 
mí, puesto que de otro modo, y sin esta síntesis, lo diverso no se unificaría en una conciencia» ${ }^{1}$ (B138). Parece, según esta afirmación del §17, que el aspecto subjetivo de la unidad —lo que necesita toda intuición para convertirse en objeto para mí- tiene que ser considerado desde o conjuntamente con el aspecto objetivo, el principio según el cual la unidad sintética de la conciencia es una condición objetiva de conocimiento. $Y$ si esto es así, de ello se sigue alguna consecuencia en cuanto a la manera de entender la deducción trascendental, en concreto, en cuanto al tipo de relación que se establece entre la primera parte y la segunda.

En el conocido §13, Kant conecta el significado de la deducción con el de justificación y legitimación del uso de nuestros conceptos. Estos dos primeros significados sitúan a la deducción en el terreno de la demostración de la legitimidad que se refiere a la pretensión de validez de ciertos conceptos, cuyo contenido puede estar relacionado de más de una manera con la experiencia. Kant menciona el procedimiento de Locke como ejemplo de una indagación que trataría de justificar la adquisición de conceptos completamente a partir de la experiencia y que por lo tanto sería una investigación empírica, para aclarar el tipo de deducción que a él le interesa como «la explicación del modo en que los conceptos a priori pueden referirse a objetos» ${ }^{2}$ (B117). Esta deducción ya no sería empírica, sino trascendental, contraposición que con ciertas oscilaciones se mantiene en todo el apartado. Tales oscilaciones se refieren al hecho de que en un primer momento Kant distingue en-

\footnotetext{
${ }^{1}$ Die synthetische Einheit des Bewusstseins ist also eine objektive Bedingung aller Erkenntnis, nicht deren ich bloss selbst bedarf, um ein Objekt zu erkennen, sondern unter der jede Anschauung stehen muss, um für mich Objekt zu werden, weil auf andere Art, und ohne diese Synthesis, das Mannigfaltige sich nicht in einem Bewusstsein vereinigen würde.

2 [...] die Erklärung der Art, wie sich Begriffe a priori auf Gegenstände beziehen können [...].
} 
tre deducción trascendental y deducción empírica en la forma que se acaba de indicar, aunque más tarde restringe el uso del término «deducción» para referirlo exclusivamente a la explicación y justificación del modo en que los conceptos priori pueden referirse a objetos. La deducción trascendental, por lo tanto, tiene en Kant un significado y un alcance mucho más restringidos que la interpretación posteriormente acuñada en el primer idealismo alemán, por ejemplo, la que expresa Fichte, cuando en Sobre el concepto de la Doctrina de la Ciencia, de 1794, menciona la necesidad de que «todo lo demostrable ha de ser demostrado -todas las proposiciones han de ser deducidas excepto el primero y supremo principio fundamental $»^{3}$ (Fichte, 1971, Bd. I: 79).

Para Kant, la deducción se mantiene dentro de los límites de la justificación de los conceptos, por lo que respecta a su validez para el conocimiento objetivo, lo que indica que el significado jurídico del término «deducción» no es simplemente trasladado al terreno epistemológico — como se ha dado a entender (ver Henrich, 1988)—, sino que también tiene sus límites en cuanto recibe una interpretación filosófica y crítica. Por ello, se va a considerar en adelante, como orientadora sobre todo, la noción de «justificación», en cuanto distinta de la de uso, pues no se trata solamente de explicar cómo se usan esos conceptos, sino con qué derecho se consideran como conceptos de objeto. Con ello, también se amplía la perspectiva de la deducción hacia el terreno de la justificación del conocimiento - hoy tan importante-, en el cual, sin duda, Kant introdujo innegables avances.

\footnotetext{
${ }^{3}$ Es muss alles Erweisbare erweisen, - ausser jenem ersten und höchsten Grundsatze müssen alle Sätze abgeleitet werden.
} 
Cuando Kant plantea a continuación la necesidad de la deducción trascendental, afirma, por una parte, que «pueden sernos dados fenómenos en la intuición sin que tengan que referirse forzosamente a las funciones del entendimiento ${ }^{4}$ (B122), pues éste no contiene las condiciones a priori para la aparición de los fenómenos. En cambio, lo que el entendimiento contiene son las «condiciones subjetivas del pensar» sin las cuales no sería posible el conocimiento de objetos. Por otra parte, Kant hace notar que la posibilidad del conocimiento de objetos exige que esas condiciones subjetivas del pensar tengan validez objetiva. En esto reside la dificultad de la deducción, o de la justificación, pues lo que es subjetivo ha de tener validez objetiva. Con esta formulación del problema queda planteada la «deducción metafísica» de las categorías y la necesidad de hallar «el hilo conductor (Leitfaden) para el descubrimiento de todos los conceptos puros del entendimiento», tal como reza el encabezamiento del §10. Según una interpretación muy extendida, se trata de llevar a cabo una indagación que en lugar de remontarse hasta las condiciones de posibilidad de la experiencia de objetos consiste en un análisis conceptual que parte de las funciones lógicas de los juicios para llegar a las formas del pensar. En esta línea de interpretación se encuentran los trabajos clásicos de Wolff (1963), Strawson (1966), y Bennett (1966), si bien, como veremos, una visión tan «lógica» de la deducción metafísica es insuficiente, puesto que no pone en claro cuál es su objetivo.

En todo caso, la necesidad de que las condiciones subjetivas del pensar tengan validez objetiva depende, a nuestro

${ }^{4}[\ldots]$ können uns allerdings Gegenstände erscheinen, ohne dass sie sich notwendig auf Funktionen des Verstandes beziehen müssen. 
entender, de dos formulaciones, incluidas en el $\S 13$, que no parecen completamente sinónimas: una es que las condiciones subjetivas del pensar «han de proporcionar las condiciones de posibilidad de todo conocimiento de objetos» (B122) $)^{5}$. Otra es que los objetos de la intuición sensible se han de «conformar, además, a las condiciones que el entendimiento exige para la unidad sintética del pensar» (B123) 6 . El pensar significa aquí el pensar de objetos, es decir, una actividad del entendimiento con respecto a una diversidad dada en la intuición. El conocimiento, en sentido amplio, viene a ser el resultado de esa actividad, toda vez que, según indica el §10, «únicamente la espontaneidad del pensar exige que esa diversidad sea primeramente recorrida, asumida y enlazada de una manera determinada, a fin de hacer de ella un conocimiento $»^{7}$ (B102). Para llevar a cabo esa actividad — llamada síntesis - el entendimiento necesita conceptos «que dan unidad a esta síntesis pura y que consisten tan sólo en la representación de esta necesaria unidad sintética ${ }^{8}$ (B104).

Se diría que Kant utiliza un argumento circular del que no se sale fácilmente, puesto que el requisito de la objetividad depende de la unidad sintética del pensar, sin la cual no es posible la actividad necesaria para el conocimiento, pero en la misma posibilidad del conocimiento esa unidad sintética ya está incluida - aunque quizá no necesariamente presupuesta en sentido estricto. La pregunta que se plantea es si efectiva-

\footnotetext{
${ }^{5}[\ldots]$ subjektive Bedingungen des Denkens sollten objektive Gültigkeit haben, d.i. Bedingungen der Möglichkeit aller Erkenntnis der Gegenstände abgeben.

${ }^{6}$ Denn dass Gegenstände der sinnlichen Anschauung [...] den Bedingungen, deren er Verstand zur synthetische Einheit des Denkens bedarf, gemäss sein müsse.

${ }^{7}$ Allein die Spontaneität unseres Denkens erfordert es, dass dieses Manningfaltige zuerst auf gewisse Weise durchgegangen, aufgenommen, und verbunden werde, um daraus eine Erkenntnis zu machen. ${ }^{8}$ Begriffe, welche dieser reinen Synthesis Einheit geben, und lediglich in der Vorstellung dieser notwendigen sysnthetischen Einheit bestehen [...].
} 
mente «el círculo de la experiencia» al que Kant se refiere en la Introducción a la primera edición de su Crítica (ver A4) exige esta argumentación circular, en la que se cuenta de antemano con que el pensar de objetos constituye una «unidad sintética». Al tema de la circularidad se volverá más adelante.

Por otro lado, la deducción trascendental muestra, en su parte objetiva, que es necesario aplicar a los objetos ciertos conceptos que contienen una síntesis necesaria. Por tanto, tiene que haber conceptos objetivos necesarios de la conformación de objetos, para que mediante ellos la percepción proporcione un objeto y llegue a ser fundamento del conocimiento. La deducción subjetiva muestra cómo «quizá pueda ser el entendimiento, a través de esos conceptos, el autor de la experiencia en la que se hallan sus objetos» ${ }^{9}$ (B127). En cierto modo, Kant está de acuerdo con Locke y con Hume, a los que menciona expresamente, en que las categorías se aplican, de hecho, a los conceptos de la experiencia. Pero esto es justamente para Kant lo que provoca la pregunta por el derecho de este uso, y lo que es más, por la justificación de su posibilidad. Tal justificación, entendida como deducción trascendental, evitaría caer en la exaltación (Schwärmerei) que según Kant propició Locke, o en el escepticismo al que se entregó Hume.

Seguidamente se nos brinda, al final del §14, una «explicación» de las categorías que ya aparece en la primera edición. Según esta explicación, las categorías «son conceptos de un objeto en general mediante el cual la intuición de éste es considerada como determinada en relación con una de las funciones lógicas del juzgar» ${ }^{10}$ (B128). Es decir, que por sí mismas las

\footnotetext{
${ }_{9}^{[}[\ldots]$ vielleicht der Verstand durch diese Begriffe selbst Urheber der Erfahrung, worin seine Gegenstände angetroffen werden, sein könne [...].

${ }^{10}$ Sie sind Begriffe von einem Gegenstande überhaupt, dadurch dessen Anschauung in Ansehung einer der logischen Funktionen zu Urteilen als bestimmt angesehen wird.
} 
categorías no nos proporcionan ningún conocimiento de objetos, pues las funciones lógicas del juzgar son meras formas de pensar objetos con las que, sin embargo, no podemos sin más conocer un objeto de una manera determinada. Para ello, tiene que producirse una intuición, que es independiente de la actividad del entendimiento y que además es sensible. De modo que los objetos de las categorías son ante todo objetos de la intuición sensible, es decir, fenómenos.

El desarrollo coherente de esta composición de elementos es un objetivo principal de la Crítica de la razón pura, desarrollo que se lleva a cabo con la exposición de las categorías y donde se muestra cómo éstas tienen que aplicarse únicamente a objetos de una intuición sensible. Este desarrollo plantea como dificultad principal la de la posibilidad misma del uso objetivo de las categorías, puesto que la intuición es independiente del entendimiento y las formas puras de la intuición son tan sólo formas de la representación de la sensibilidad, por lo tanto independientes también de la espontaneidad del entendimiento. Dicho de otro modo, cómo es posible que haya correspondencia entre la intuición sensible de un objeto y los conceptos puros del entendimiento ${ }^{11}$. Si a esto se añade que los conceptos puros o categorías son también considera-

\footnotetext{
${ }^{11}$ También se aclara esta cuestión manejando una interpretación de la «deducción metafísica» distinta de la que se ha mencionado. Si tenemos en cuenta que en la $2^{\text {a }}$ edición de la Crítica, la «deducción metafísica» se refiere expresamente a la exposición de las formas de espacio y tiempo, se puede obtener una aclaración de su alcance recurriendo a la estética trascendental, por analogía con la distinción que allí hace Kant entre exposición metafísica y exposición trascendental. En consecuencia, cabe considerar a la deducción metafísica como un análisis de las condiciones que tienen que cumplirse si se quiere afirmar que conceptos a priori pueden referirse a objetos. Este análisis comprendería tres fases: 1) Aclarar bajo qué condiciones es posible esto, lo que implica explicar el significado de «categoría». 2) Mostrar que es a la función unificadora del entendimiento a la que se puede retrotraer la posibilidad de esa referencia de los conceptos a priori. 3) Señalar qué conceptos son los que cumplen las condiciones de 1) y que pueden ser considerados como instrumentos de la función unificadora del entendimiento. Sólo sobre la base de esta aclaración metafísica se puede pasar a la deducción trascendental, (ver Horstmann, 1984: 15-33).
} 
dos como «acciones del pensar puro» (Handlungen des reinen Denkens, A57/B81), y como «funciones»(Funktionen, B122) cabe afirmar que del propio texto kantiano se pueden obtener interpretaciones claramente diferenciadas, según se acentúe la característica de las categorías como «formas», como «funciones» $\mathrm{O}$ «acciones» del pensar. Esta triple faceta se hace presente en la cuestión de la correspondencia antes mencionada, cuya posibilidad es lo que pone de manifiesto no ya la deducción trascendental, sino las condiciones que la hacen posible y que además dan cuenta de su necesidad.

Enlace y unidad de la conciencia

La reelaboración de la deducción trascendental para la segunda edición abre la sección segunda, con el §15, sobre la «posibilidad de una combinación (Verbindung) en general» ${ }^{12}$. En realidad, esta reelaboración comienza ya al final de la sección primera (B127-129), con los párrafos añadidos sobre Locke y Hume a los que se acaba de hacer referencia. Bajo este supuesto, esas adiciones muy posiblemente no se limitan únicamente a ampliar el §14, sino que poseen también un significado sistemático en relación con los siguientes parágrafos de la segunda edición, los cuales se pueden interpretar también como una respuesta a Locke y a Hume, esto es, a las dificultades del empirismo.

Todo el §15 está dedicado a exponer el concepto de Verbindung o conjunctio. Hay que señalar que esta exposición en

\footnotetext{
12 El término alemán «Verbindung» ha sido traducido como «combinación» en I. Kant, 1978. También la traducción inglesa de W.S. Pluhar utiliza «combination» (ver I. Kant, 1996). Nosotros utilizaremos el término «conjunctio» y también «conjunción» y «enlace», según el contexto.
} 
general va unida precisamente a las condiciones bajo las cuales tal «conjunctio» es posible, como indica el subtítulo de dicho parágrafo. Pues bien, el enlace de algo diverso sólo puede llevarse a cabo mediante un acto del entendimiento, lo que de momento quiere decir que si yo tengo ante mí una diversidad unida, es que tengo esa «conjunctio», que está contenida en mi representación de lo diverso, y la tengo antes de que se produzca esa representación. Esto presupone que yo tengo representaciones diversas que representan objetos, las cuales son, ante todo, determinaciones subjetivas, es decir, de mi ánimo (Gemüt), de suerte que, para que representen un objeto tiene que llegarles algo por medio de lo cual se convierten en determinaciones de objetos. Kant subraya que la conjunción de una diversidad en general «nunca puede llegar a nosotros a través de los sentidos, y por tanto tampoco puede estar contenida a la vez en la forma pura de la intuición sensible; pues es un acto de la espontaneidad de la facultad de la representación $[\ldots]$ (... $(\mathrm{B} 130)^{13}$. Esto es, la representación de una serie conjunta de representaciones diversas que son determinaciones de objetos tiene que provenir de la espontaneidad de la facultad de representar, pues tal conjunción no está contenida en las representaciones que de por sí son determinaciones subjetivas. Por lo tanto, el concepto de objeto consiste en una representación que relaciona diversas representaciones dadas con lo que se representa mediante ellas. La representación de la copertenencia de varias representaciones en una única representación es la representación de una unidad, que no está dada, sino que se efectúa activamente, y que es justamente la representación del

\footnotetext{
${ }^{13}$ Allein die Verbindung (conjunctio), eines Mannigfaltigen überhaupt, kann niemals durch Sinne in uns kommen, und kann also auch nicht in du reinen Form der sinnlichen Anschauung zugleich mit enthalten sein denn sie ist ein Actus der Spontaneität der Vorstellungskraft [...].
} 
vínculo de todas esas representaciones mediante un acto de la espontaneidad. Se trata de la representación de esa conjunctio o Verbindung, que es una condición para que nos representemos objetos por medio de lo diverso de las determinaciones subjetivas - a las que también hay que llamar representaciones-. Consiguientemente, la conjunctio es una representación solamente posible a través del entendimiento, ya sea que la diversidad de la intuición sensible venga dada a través de los sentidos, o que esté contenida en las formas a priori de la intuición.

Por otra parte, la acción intelectual de conjuntar o enlazar se aplica a conceptos cuya conjunción se representa en el juicio. Sobre esta identidad de la función del entendimiento en estos dos actos de enlazar se funda la deducción de las categorías, pero con esto no se aclara dónde se funda la concordancia entre el contenido de la intuición y la forma del juicio, puesto que según el §14 el fundamento de la conjunción de los conceptos en el juicio tiene que ser también el fundamento de su concordancia.

Por el momento, la conjunción en cuanto acto de enlace, es una acción del entendimiento que se aplica a intuiciones sensibles, o «no sensibles» $\left(\right.$ B130 ${ }^{14}$. Con esta indicación, Kant introduce un problema, ya que el entendimiento no tiene por sí mismo la diversidad de la intuición, esto es, no la produce, lo que significa que el hecho de tener una representación no implica la existencia del objeto de la misma. Por eso, el entendimiento es la facultad de enlazar y de separar la diversidad dada en la intuición. Y la intuición es sensible «porque — como se lee al final de la «Estética trascendental»— no es originaria,

\footnotetext{
${ }^{14}$ [...] so ist alle Verbindung [...], es mag eine Verbindung des Mannigfaltigen der Anschauung, oder mancherlei Begriffe, und an der ersteren der sinnlichen, oder nicht sinnlichen Anschauung sein, eine Verstandhandlung [...].
} 
es decir, porque no es de tal índole que se nos dé mediante ella la existencia misma del objeto de la intuición [...], sino que depende de la existencia del objeto, con lo cual sólo es posible en tanto que la facultad de representación del sujeto es afectada por dicho objeto» (B72) ${ }^{15}$. Así que no sabemos cómo, en estas condiciones, pudiera haber una intuición no sensible, ni tampoco sabemos si, en el caso de que la hubiera, haría falta un acto de enlace además del acto en que se nos daría el objeto con todo su contenido.

Lo que se establece taxativamente es que no conocemos nada a través de una intuición intelectual, pues no somos capaces de tenerla. Y también, que la acción de enlazar lo diverso, más que referirse a la intuición sensible en especial, se refiere propiamente a la intuición en general aunque, si bien se mira, la concepción kantiana de esta conjunción o enlace (Verbindung) está referida específicamente a una diversidad dada y, por lo tanto, faltando ésta, el acto de la conjunctio quedaría vacío. Es claro que podemos pensar en la posibilidad de un acto como éste, pero la conciencia de tal acto no sería una conciencia de objeto. Por lo tanto, cuando el pensar se refiere a algún objeto, es que hay alguna intuición, es decir, una representación inmediata de ese objeto en la cual se da. El hecho de que no podamos tener una intuición no sensible, pues toda posibilidad de representarnos un objeto está condicionada por las formas de la intuición, que son a priori, quiere decir que nuestra intuición está subjetivamente condicionada y, por ende, que es sensible. Parece entonces, más bien, que Kant deja abierta la capacidad del entendimiento en cuanto facultad de

${ }^{15}[\ldots]$ weil sie ist nicht ursprünglich, d. i., eine solche ist, durch die selbst das Dasein des Objekts der Anschauung gegeben wird [...], sondern von dem Dasein des Objekts abhängig, mithin nur dadurch, dass die Vorstellungsfähigkeit des Subjekts durch dasselbe affiziert wird, möglich ist. 
enlazar lo diverso de la intuición y que la limitación viene del condicionamiento de la conciencia humana, precisamente en cuanto conciencia representativa.

Concretando más el significado de Verbindung, Kant añade que «es, entre todas las representaciones, la única que no viene dada mediante objetos, sino que, al ser un acto de la espontaneidad (Selbsttätigkeit) del sujeto, sólo puede ser realizada por éste» $(B 130)^{16}$. Por lo tanto, se trata de un acto que pone de relieve la capacidad activa del sujeto. De esto se desprende que un objeto no es de por sí una conjunción de partes, sino que la conjunción se sigue de mi acto de enlazar o, dicho de otro modo, que es un producto de mi entendimiento. Así, se pone de manifiesto una íntima conexión entre conocer y hacer, característica del planteamiento epistemológico kantiano (ver Baum, 1984). El entendimiento en su actividad de enlazar no está condicionado por el material, dado, sino que es pura espontaneidad y su actividad es por eso, literalmente, autoactividad. Ahora bien, si no hay material, esa actividad se queda vacía, con lo cual cabe decir que aunque no haya condicionamiento, la actividad de enlazar está referida a algo dado, sobre la cual recae la conjunctio. Pero cuando hay intuición sensible, intuición que es dependiente de la existencia de un objeto, la actividad del entendimiento se desarrolla sin condicionamientos extrínsecos a él, espontáneamente, y es por el contrario el objeto, o más exactamente lo que Kant llama objeto, el que depende de esa actividad.

Además, este acto de enlazar, al que Kant se refiere con la designación general de sintesis, «tiene que ser originariamente

\footnotetext{
${ }^{16}$ [...] unter allen Vorstellungen die Verbindung die einzige ist, die nicht durch Objekte gegeben, sondern nur vom Subjekte selbst verrichtet werden kann, weil sie ein Actus seiner Selbsttätigkeit ist.
} 
uno, indistintamente válido para toda combinación ${ }^{17}$ (B130). Pero, como hemos visto, la conjunción se tiene que ejercer sobre un material sensible; por lo cual, a la hora de calificar a este tipo de síntesis, hay que señalar que no se trata de una síntesis «en general», sino más bien de una clase de síntesis muy particular: la que combina una diversidad dada en la intuición, para la cual no tenemos ningún concepto determinado. Y como toda intuición está condicionada espacial y temporalmente, se tratará de una síntesis concreta, «figurada" y no de una síntesis intelectual propiamente dicha. Por lo demás, el que sea un acto único y válido por igual para toda conjunctio puede indicar también que se trata únicamente de una función unificadora sin más.

El último párrafo del §15 se refiere en primer lugar a esa función, puesto que este enlace «es la representación de la unidad sintética de lo diverso» ${ }^{18}$ (B131). Es decir, por una parte, la unidad no surge de la conjunctio sino que más bien la hace posible y, por otra parte, lo diverso tampoco es una simple pluralidad de elementos, o de cualidades. Por ello, la separación de la facultad de enlazar como tal frente a lo que enlaza necesita alguna explicación más, sobre todo teniendo en cuenta que esa unidad sintética no es una categoría, ya que la categoría también presupone la conjunctio.

Recordemos que según el método kantiano de exposición (Darstellung), la deducción debe probar la validez objetiva de las categorías respecto al conocimiento, y por eso Kant analiza la posibilidad de que el entendimiento sea una facultad cognoscitiva. Esta investigación que, por un lado avanza sintéti-

\footnotetext{
${ }^{17}$ Man wird hier leicht gewahr, dass diese Handlung ursprünglich einig, und für alle Verbindung gelichgelten sein müsse [...].

${ }^{18}$ Verbindung ist Vorstellung der synthetischen Einheit des Mannigfaltigen.
} 
camente, es decir, de las condiciones a lo condicionado, tiene su introducción en el §15. Pero por otro lado, según el procedimiento analítico tiene que ir también de lo condicionado - la conjunctio - a sus condiciones, es decir, a la síntesis de su condición, que es la unidad sintética originaria. Kant realiza este doble itinerario, puesto que el procedimiento analítico requiere asimismo el sintético, como se puede apreciar en el $\S 15$, donde trata conjuntamente de la síntesis de los conceptos en el juicio y de la síntesis de lo diverso en la intuición, aspectos que luego separa en los parágrafos siguientes hasta cerrar el desarrollo en el §26.

También hay que tener en cuenta las condiciones de posibilidad de los enlaces o de las acciones de enlazar, que Kant considera como actos que tienen que haber tenido lugar previamente, puesto que «no podemos representarnos nada como enlazado en el objeto sin haberlo tenido enlazado antes» ${ }^{19}$ (B130). La acción del análisis presupone en cada caso la del enlace o conjunción, «pues donde el entendimiento no ha enlazado nada antes, nada puede él tampoco disolver $»^{20}$ (B130). Esta progresión temporal está tomada de la conexión empírica de las representaciones, de la cual podemos ser conscientes en nuestra experiencia interna. Así que la síntesis figurada ha tenido lugar ya, seamos o no conscientes de ello; esa síntesis es una actividad que se ejerce sobre el material sensible dado.

Sobre el significado general de «síntesis», en el §10 se encuentran dos afirmaciones que nos parecen muy esclarecedoras. Por un lado, afirma Kant que «la síntesis de algo diverso... produce ante todo un conocimiento que, inicialmente, puede

\footnotetext{
${ }^{19}[\ldots]$ wir uns nichts, als im Objekt verbunden, vorstellen können, ohne es vorher selbst verbunden zu haben $[. .$.$] .$

${ }^{20}[. .$.$] denn wo der Verstand vorher nichts verbunden hat, da kann er auch nichts auflösen [...].$
} 
ser todavía tosco y confuso y que, por ello mismo, necesita un análisis» ${ }^{21}$ (B103). Por otro lado, señala que «la síntesis es un mero efecto de la imaginación, una función ciega, si bien indispensable, del alma, sin la cual no tendríamos conocimiento alguno y de la cual sin embargo rara vez somos conscientes ${ }^{22}$ (B103). Por lo tanto, el presupuesto trascendental de la conjunctio como acción inconsciente de síntesis está ya presente, toda vez que el método analítico presupone, de hecho, una conjunctio que puede ser realizada sobre el material empírico y que por tanto pertenece simplemente a la actividad del alma. Pero además, la síntesis de la imaginación tiene que llamarse también trascendental, pues de ella se sigue la posibilidad del conocimiento, es decir, de lo que resulta enlazado o conjuntado.

Con estas precisiones volvemos al §15, según el cual la representación de la conjunctio es posible porque la lleva a cabo el entendimiento. Como vimos también, una conjunctio o Verbindung se define como la representación de la unidad sintética de lo diverso (ver B131). Y puesto que la síntesis de lo diverso tiene en su base una unidad sintética, parece que esta unidad surge del acto de conjuntar, si bien el concepto de conjunctio contiene ya el concepto de unidad, como su condición lógica. Por lo demás, como este tipo de enlace sólo puede ocurrir mediante una acción de la espontaneidad de la facultad de representación, lo que posibilita tal enlace es la unidad a priori previamente representada de una diversidad no unida. De modo que la representación a priori de la unidad como

\footnotetext{
${ }^{21}$ Die Sintesis eines Mannigfaltigen aber [...] bringt zuerst eine Erkenntnis hervor, die zwar anfänglich noch roh oder verworren sein kann, und also der Analysis bedarf.

${ }^{22}$ Die Synthesis überhaupt ist [...] die blosse Wirkung der Einbildungskraft, einer blinden, obgleich unentbehrlichen Funktion der Seele, ohne die wir überall gar keine Erkenntnis haben würden, der wir uns aber selten nur einmal bewusst sind.
} 
principio de la síntesis hace posible la acción de conjuntar como tal y también el concepto mismo de conjunctio. De nuevo aparece una relación de circularidad entre Verbindung y unidad sintética, que da razón de un aspecto fundamental de la exposición kantiana.

Se mencionó anteriormente que la categoría presupone la conjunctio. En efecto, la unidad que se funda en la síntesis de representaciones es a su vez una representación que precede a todos los conceptos particulares de conjunctio, y por eso esta unidad no es propiamente un concepto, al modo como lo son las categorías correspondientes (unidad, pluralidad, totalidad). Pues por un lado el concepto de unidad no puede ser una representación, y por otro lado las categorías son de suyo formas de enlace de lo diverso y presuponen, en cuanto tales, la representación de la unidad, que es condición lógica de la posibilidad de las mismas. Dicho de otro modo, la unidad pensada en las categorías surge de la aplicación de las funciones del juicio a la intuición. Por consiguiente, si buscamos la condición de posibilidad de toda conjunción, tendremos que ir al fundamento de esa posibilidad, que debe estar en un nivel más alto que el de las formas del juicio. En resumen, tendremos que ir de los conceptos de enlace relacionados con las formas de la intuición a las funciones del juicio y de éstas al fundamento de su posibilidad. En él tiene que fundarse en última instancia la unidad sintética de las intuiciones que es pensada en una categoría. Él tiene que ser también el fundamento de la concordancia de la diversidad de la intuición con las funciones del juicio, pues lleva a cabo la conjunción de lo diverso que luego se completa en esas funciones.

Consiguientemente, habría que preguntar cuál es ese fundamento último de unidad, cómo fundamenta las funciones del juicio y cómo produce esa concordancia entre los en- 
laces y dichas funciones. Por lo pronto, sabemos sólo que de alguna manera tiene que hacer posible la conjunción en cuanto unidad sintética, así que tiene que ser él mismo una unidad sintética, pero originaria, es decir, que sirva de fundamento a toda conjunción posible y que él mismo no sea algo unificado y puesto. También tendrá que ser fundamento de la unidad sintética de la conciencia de las diversas representaciones, ya sean intuiciones o conceptos, y tendrá que determinar esa síntesis de la conciencia. Y finalmente tendrá que hacer posible la unidad sintética de los conceptos pensados en el juicio. El final del $\S 15$ introduce de algún modo esta cuestión del fundamento, al menos como presupuesto del camino que permite regresar a la condición última de toda conjunctio.

En esas líneas finales, aparecen dos características de la unidad que se ha de buscar. Una es la que tiene que ver con su aspecto cualitativo, es decir, que la unidad suprema que sirve de fundamento a toda conjunctio posible es una unidad cualitativa y no puede ser de otra manera. Pues no se trata de una unidad resultante de reunir diversas partes en un todo, sino de una unidad que es ya en sí misma un todo, y un todo único en cuanto es el que funda toda unidad sintética posible. La síntesis que surge de la misma es única, como dicha unidad, y originaria, pues proporciona los diversos principios de las diversas síntesis kantianas. Pero esta unidad cualitativa que está presupuesta en toda conjunctio no se refiere a una cualidad determinada, sino que es una cualidad formal, en cuanto unidad suprema del fundamento común de todas las conjunciones de diversidades cualitativamente distintas. Otra característica se refiere a algo que Kant indica muy brevemente, cuando escribe que tenemos que buscar esa unidad incluso en aquello mismo que contiene «el fundamento de posibilidad del en- 
tendimiento, incluso en su uso lógico ${ }^{23}$ (B131). Con este final del parágrafo dedicado a la conjunctio, Kant nos quiere hacer recordar todo lo que ha expuesto en la presentación de la tabla de las categorías, y en concreto que los conceptos puros del entendimiento forman una tabla trascendental de categorías puesto que se refieren de modo a priori a objetos ${ }^{24}$. Pues bien, como las categorías presuponen el enlace que es pensado en los juicios y como la facultad de enlazar fundamenta también el conocimiento de los objetos de esos juicios — conocimiento cuya posibilidad se investiga de un modo trascendental -, por eso mismo la unidad sintética que buscamos es una unidad trascendental.

Hasta ahora esta unidad se ha considerado solamente de un modo analítico, sin considerar en qué consiste esta unidad sintética y de dónde viene el que sea necesariamente ella la que sirva de fundamento a la conjunción de todas las representaciones, que es lo que trata Kant seguidamente.

\section{El principio trascendental de la apercepción}

Es sobradamente conocido el comienzo del §16: «El Yo pienso tiene que poder acompañar todas mis representaciones; pues si no sería representado en mí algo que en absoluto podría ser pensado, lo que viene a querer decir que la representación o

\footnotetext{
${ }^{23}$ Also müssen wir diese Einheit $[\ldots]$ noch höher suchen, nämlich in demjenigen, was selbst den Grund der Einheit verschiedener Begriffe in Urteilen, mithin der Möglichkeit des Verstandes, sogar in seinem logischen Gebrauche, enthält.

${ }^{24}$ Para Röttges, la dialéctica entre significado y uso, que opera en toda la Analítica, es lo que permite distinguir entre «a priori» $\mathrm{y}$ «trascendental». Porque si significado y uso de las formas de conocimiento fueran idénticos, también serían indistinguibles «a priori» $\mathrm{y}$ «trascendental». Con otras palabras, si las formas de conocimiento tienen un significado para sí, sin uso, lo a priori no se puede determinar como trascendental, recurriendo a las condiciones de posibilidad de la experiencia. Ver Röttges, 1981: 49 .
} 
bien sería imposible o al menos no sería nada para mí» ${ }^{25}$ (B132). Por lo tanto, la conciencia de que yo tengo una representación cualquiera tiene que ser siempre posible, y esto es una proposición analítica o idéntica, además de necesaria. Esto no quiere decir que siempre soy consciente de todas mis representaciones, cosa que de hecho no ocurre, sino que yo tengo que poder ser consciente de todas aquellas representaciones a las que yo me refiero como «mis representaciones». Hay una conjunción originaria (ursprüngliche Verbindung, B133) entre estos dos términos, yo y representación, que consiste en la pertenencia de una representación a mi yo como facultad de representarme algo, de suerte que eso es justamente lo que quiere decir «mi representación», y el acto de que se me haga consciente la misma es la realización de lo que eso significa. Esto apunta, por lo pronto, al carácter interno de las representaciones, en el sentido de que sólo yo me percato de ellas, y en cambio otro, es decir, alguien que estuviera en una relación externa con respecto a la representación, no puede percatarse de ellas. Sin embargo, no es sólo ni principalmente esta conexión interna lo que caracteriza a la vinculación del yo pienso con mis representaciones, pues este tipo de análisis debe ser abandonado de inmediato para comprender la verdadera índole del yo pienso.

Con todo, este análisis sirve también para destacar, en comparación con la intuición sensible, otra característica del yo pienso. La intuición sensible es una diversidad que guarda una relación necesaria con la conciencia de mi pensar incluso antes de que ese pensar se ejercite sobre ella, es decir, antes de que la intuición sea unida a un concepto determinado. Am-

\footnotetext{
${ }^{25}$ Das: Ich denke, muss alle meine Vorstellungen begleiten können; denn sonst würde etwas in mir vorgestellt werden, was gar nicht gedacht werden könnte, welches eben so viel heisst, als die Vorstellung würde entweder unmöglich, oder wenigstens für mich nichts sein.
} 
bos, el pensar y lo diverso de la intuición, le son presentes a un sujeto, pero las representaciones de la intuición sensible le vienen de fuera, lo que quiere decir que se deben a una afección. En cambio, la representación del propio pensar que se expresa con el yo pienso se lleva a cabo mediante un acto espontáneo del sujeto pensante. Esto quiere decir que la representación del propio pensar es también un pensar, y no una intuición, por lo tanto que no es una afección que viene de algo ajeno. Con otras palabras, la conciencia de mí mismo que expreso con el «yo» del yo pienso es el acto del pensamiento de representarme a mí mismo como pensante. Esta falta de determinación del yo como sujeto del pensamiento con respecto a lo que son de suyo las representaciones concretas es una consecuencia que Kant caracteriza en los «Paralogismos de la razón pura» como «un yo que designa [...] un sujeto lógicamente simple» ${ }^{26}$ (B407).

Acerca de esta singular representación, cabe decir que el yo pienso es la autoconciencia, la cual consiste en una apercepción o apperceptio, y no en una perceptio, como es cualquier representación consciente, aunque ya en el adjetivo «consciente» se encierra la referencia a mi yo autoconsciente. La necesaria distinción entre el yo de la percepción y el yo de la apercepción permite distinguir entre el plano empírico y el plano trascendental, así como entender mejor la noción de autoconciencia. Porque yo no tengo tan sólo la representación de que todas las representaciones son determinaciones mías, o de mi facultad de representación, sino que tengo la representación de aquello que caracteriza a la conciencia misma, a toda conciencia, es decir, la condición de toda representación posible. El yo que es sujeto del pensamiento no es un yo individual, y por

${ }^{26}[\ldots]$ das Ich der Apperzeption [...] mithin ein logisch einfaches Subjekt bezeichne [...]. 
lo mismo no puede transformarse en una pluralidad, pues es el yo singular en cuanto sujeto del pensar. Y podríamos decir también que la conciencia empírica, que es conciencia de alguna representación, depende de la autoconciencia pura, que es estrictamente apercepción. Por lo demás, el yo pienso como tal no es un yo aislado del pensar, sino que está unido al ejercicio de esta actividad. Lo que piensa este yo es el pensamiento yo pienso como sujeto de todas mis representaciones. El yo como sujeto del pensar que sirve de base a toda representación es por ello un pensamiento originario. Esta característica de originariedad la destacó Hegel tempranamente, en Glauben und Wissen (1802), viendo en la unidad sintética de la apercepción la verdadera idea de la filosofía (ver Hegel, 1968: GW 4, 327).

Por ello, el carácter originario de la apercepción se tiene que explicar no sólo porque el yo pienso no padece ninguna afección —eso sería una explicación insuficiente- sino porque las representaciones particulares de que yo pienso esto o lo otro, en las cuales está incluido el acompañamiento del yo pienso, sólo pueden ser pensadas gracias a un acto que no surge de una representación u otra, sino de mí mismo, que en cuanto sujeto del pensamiento es, por así decirlo, el acompañante. El yo pienso es el mismo en cualquier tipo de pensamiento, precisamente porque el poder acompañar depende sólo del sujeto pensante. Por consiguiente, la autoconciencia de este sujeto pensante no puede ser acompañada de algo que es sólo una representación, sino que es ella misma la acompañante.

A esta capacidad originaria del yo pienso para acompañar todas las representaciones de un sujeto está vinculada la identidad de la conciencia de sí. Porque, si afirmamos que la autoconciencia es una y la misma en toda conciencia, queremos decir, por un lado, que se trata de la identidad de la representación de mí mismo en cuanto sujeto o ser pensante, y también 
queremos decir, por otro lado, que esta autoconciencia posee o es una unidad trascendental pues el principio de que «el yo pienso tiene que poder acompañar mis representaciones» es lo que funda a priori la posibilidad del conocimiento. Con otras palabras, la apercepción es «el entendimiento mismo» (B134n), como dice Kant en nota en el §16, ya que tal apercepción hace posible todo lo que el entendimiento puede hacer. Todos los conceptos comunes, afirma en esa nota, remiten a la unidad analítica de la conciencia. Por lo tanto, mi conciencia de esas representaciones está en relación necesaria con una autoconciencia y yo debo ser consciente de que soy yo quien piensa esas representaciones. Esta autoconciencia - común a todas ellas - es la condición bajo la cual se encuentran todas las representaciones que tengo, las cuales están unidas de un modo originario, y no mediante una unión de hecho, puesto que se trata de una unión determinante en cuanto soy el sujeto de todas ellas. Así, sigue diciendo Kant en esa nota que la unidad de la apercepción «es el punto más elevado del que ha de depender todo uso del entendimiento, incluida la Lógica entera ${ }^{27}$ (B134).

Siendo esto así, la identidad de la apercepción debe ser algo más que el acompañamiento y la pertenencia de las representaciones conscientes a una misma conciencia. En efecto, Kant escribe que «sólo porque puedo enlazar en una conciencia la diversidad dada de las representaciones, es posible que yo me represente la identidad de la conciencia en esas representaciones mismas, es decir, la unidad analítica de la apercepción es posible sólo bajo el presupuesto de cierta unidad sintética» ${ }^{28}$

\footnotetext{
${ }^{27}$ Und so ist die synthetische Einheit der Apperzeption der höchste Punkt, an dem man allen Verstandesgebrauch, selbst die ganze Logik, $[\ldots]$ heften muss [...].

${ }^{28}$ Also nur dadurch, dass ich ein Mannigfaltiges gegebener Vorstellungen in einem Bewusstsein verbinden kann, ist es möglich, dass ich mir die Identität des Bewusstseins in diesen Vorstellungen
} 
(B133). Por consiguiente, la autoconciencia ha de ser la representación espontánea de un pensar que hace posible todos los conceptos; y la necesaria cohesión de lo diverso no puede venir dada, sino que tiene que provenir de una actividad de poner. Asimismo, la conexión de la conciencia de lo diverso en la autoconciencia es una conexión originaria. Así que en la conciencia de esta conexión se funda la conciencia que es necesariamente posible, es decir, la conciencia de la identidad numérica de todas las conciencias, por muchos actos de conciencia singulares que se lleven a cabo.

Por otra parte, que la unidad analítica de la apercepción sea posible sólo bajo el presupuesto de cierta unidad sintética supone que la representación de la conciencia idéntica en todas mis representaciones es la representación de algo que puede ser unido en cada ocasión, pero igualmente también que puede ser aislado del contenido de la conciencia mediante la abstracción y la reflexión del pensar sobre sí mismo. De modo que la representación de «yo pienso esto», que es una conciencia determinada, se convierte en la representación «yo pienso» por medio del análisis y esta conciencia de mí mismo es la unidad analítica de la autoconciencia que tiene que poder acompañar todas mis representaciones. Este análisis consiste en la disolución del vínculo entre el yo pienso y la representación y de él cabe decir también lo que Kant dice del análisis de la conjunctio, que aunque parece ser lo contrario de la síntesis, en realidad la presupone. Esto se puede afirmar siempre y cuando aceptemos que el análisis se dirige no tanto a la representación, cuanto a la conciencia de la identidad de la autoconciencia, de modo que yo puedo representarme el yo

selbst orstelle, d. i. die analytische Einheit der Apperzeption ist nur unter der Voraussetzung irgend einer synthetischen möglich. 
pienso como idéntico sólo si a la vez considero la separación de la conciencia empírica y me hago consciente de la síntesis de esa o de cualquier otra conciencia posible. En este sentido, se puede ver que la unidad analítica de la apercepción sólo es posible bajo el presupuesto de la unidad sintética.

Esta relación entre unidad analítica y unidad sintética se encuentra también con respecto a la conciencia de las representaciones. La unidad analítica de la conciencia es inherente a todos los conceptos comunes en cuanto tales. Es decir, la representación de una propiedad o característica común de varias cosas se obtiene por análisis de la representación conjunta de la cosa, en la cual hay una conjunctio de representaciones, y esa representación conjunta a su vez la tenemos gracias a una síntesis que previamente nos ha proporcionado el contenido total. Ahora bien, la unidad sintética que hace posible tener una representación de un conjunto de características tiene que haber sido previamente pensada, para poder llegar luego a una unidad analítica. En otros términos, esto se puede interpretar en el sentido de que el pensamiento de la unidad requiere y supone, en este contexto al menos, el pensamiento de la diferencia. Este pensamiento de la diferencia es una síntesis que el sujeto efectúa, no es algo encontrado previamente, sino algo realizado por el sujeto pensante. La condición de esta síntesis es, una vez más, la unidad sintética originaria. Y como los conceptos comunes son unidades analíticas de la conciencia de una diversidad, estos conceptos, o dicho de otro modo, la conciencia de una parte idéntica de diversas conjunciones de representaciones, tiene que fundarse en la conciencia de la conjunción de esa diversidad, que es la que puede ser analizada. Así, señala Kant que «la síntesis es lo primero a lo que 
tenemos que atender si queremos juzgar sobre el origen primero de nuestro conocimiento ${ }^{29}$ (B103).

Por consiguiente, un determinado concepto es general sólo bajo el supuesto de una conciencia de la síntesis de representaciones dadas, que es una conciencia de mí mismo, o conciencia de mi idéntico yo pienso. Ahora bien, como pensar es unificar (vereinigen) representaciones en una conciencia, la conciencia de mi yo idéntico en toda conciencia concreta no es la conciencia de una cosa a la que están referidos contenidos representativos, sino la conciencia de un acto que tiene que llevarse a cabo cada vez que se me da un material representado.

El carácter no empírico de esta autoconciencia y de su actividad queda subrayado al considerar el yo pienso como el fundamento trascendental de la unidad de la conciencia. Esto representa un nivel superior de la relación entre el yo y sus representaciones, ya que es esta dimensión la que da lugar a la relación originaria del sujeto cognoscente con el objeto puesto ante él ${ }^{30}$. La distinción entre apercepción empírica y apercepción trascendental permite aclarar el significado de dicha relación, así como caracterizar de un modo más preciso a la subjetividad. Aunque, en realidad, como se ha venido mostrando, para Kant el yo pienso sólo cabe entenderlo en sentido trascendental, ya que «la conciencia empírica que acompaña representaciones diversas es en sí misma dispersa

\footnotetext{
$29 \quad[\ldots]$ sie [die Sintesis] ist also das erste, worauf wir Acht zu geben haben, wenn wir über den ersten Ursprung unserer Erkenntnis urteilen wollen.

30 No obstante el énfasis de Kant en el carácter objetivo de la relación entre el «yo pienso» y mis representaciones - ya se articulen como conceptos o como juicios - , Hegel considera en el escrito anteriormente citado que el análisis kantiano transforma el idealismo trascendental en un idealismo formal, o más exactamente psicológico, al considerar la unidad del «yo pienso» como unidad de la autoconciencia de un sujeto que tiene experiencia y de la cual en todo caso depende. Ver Glauben und Wissen, en GW 4, 331.
} 
y carece de relación con la identidad del sujeto» ${ }^{31}$ (B133). Por ello, es necesario que toda conciencia empírica esté en relación con una conciencia trascendental, es decir, con la experiencia de mi yo como apercepción originaria. Esto entraña a la vez que el vínculo entre el yo pienso y sus representaciones es una relación objetivamente válida, además de necesaria, completamente distinta del vínculo subjetivo entre la conciencia empírica y el flujo de sus determinaciones internas (ver A 117; nota).

Por último, Kant llama condición trascendental de todo pensar a la unidad sintética de la apercepción, que es unidad objetiva de la autoconciencia (ver B139) y, en cuanto tal, la posibilidad de la forma lógica de todo conocimiento, de todos los juicios. Con ello se explicita la relación de los conceptos en el juicio con la apercepción originaria. Esta relación no depende del contenido de los conceptos, sino de la necesaria unidad de la apercepción, en virtud de la cual las representaciones que pueden constituir algún conocimiento quedan determinadas objetivamente. Así, cuando Kant afirma que un juicio es una relación objetivamente válida entre representaciones, a diferencia de la relación psicológica y por tanto subjetiva de las mismas en la conciencia empírica, está destacando el carácter trascendental del yo pienso y la imposibilidad de fijarlo en términos empíricos.

$31 \quad[\ldots]$ das empirische Bewusstsein, welches verschiedene Vorstellungen begleitet, ist an sich zerstreut und ohne Bezeihung auf die Identität des Subjekts. 


\section{BIBLIOGRAFÍA}

Baum, M. (1984). Erkennen und Machen in der «Kritik der reinen Vernunft». En B. Tuschling (ed.), Probleme der «Kritik der reinen Vernunft». Berlin: W. De Gruyter.

Baumgartner, H. M. (1985). Kants «Kritik der reinen Vernuft». Einleitung zur Lektüre. Freiburg: Alber.

Becker, W. (1984). Selbstbewusstsein und Erfahrung. Zu Kants tranzendentaler Deduktion und ihrer argumentativen Rekonstruktion. Freiburg: K. Alber.

Bennett, J. (1966). Kant's Analytic. Nueva York: Cambridge University Press.

Bossart, W. H. (1994). Apperception, Knowledge, and Experience. Ottawa: University of Ottawa Press.

Cramer, C. (1985). Nicht-reine synthetische Urteile a priori. Ein Problem der Transzendentaphilosophie Immanuel Kants. Heidelberg: C. Winter.

Duque, F. (2002). La fuerza de la razón: invitación a la lectura de la «Crítica de la razón pura». Madrid: Dykinson.

Fichte, J. G. (1971). Über den Begriff der Wissenschaftslehre, §7. I. H. Fichte (ed.) Sämtliche Werke, 1834-46, Band I. Berlin: Walter de Gruyter, 79.

Gerlach, H.-M., Moecke, S., (eds.) (1982). Kants «Kritik der reinen Vernuft» imphilosophischen Meinungsstreit der Gegenwart, Halle/Saale: Martin-Luther-Universität Halle-Wittenberg.

Gurwitsch, A. (1990). Kants Theorie des Verstandes. T.M. Seebohm (ed.). Dordrecht: Kluwer.

Hegel, G.W.F. (1968). Glauben und Wissen oder die Reflexionsphilosophie der Subjectivität, in der Vollständiger 
ihrer Formen, als Kantische, Jacobische und Fichtesche Philosophie. Gesammelte Werke (GW) Bd. 4. Jenaer Kritische Schriften,. H.Buchner \& O. Pöggeler (eds.). Hamburg, GW, 4, 315-414.

Heidegger, M. (1991). Kant und das Problem der Metaphysik. $5^{\text {a }}$ ed., Frankfurt/Main: Klostermann.

Heidegger, M. (1987). Phänomenologishe Interpretation von Kants «Kritik der reinen Vernuft». Frankfurt/Main: Klostermann.

Henrich, D. (1988). Kants transzendentale Deduktion und die Möglichkeit von Transzendentalphilosophie. Homburg B. (ed.), Forum für Philosophie. Frankfurt/Main: Suhrkamp.

Homburg, B. (ed.) (1988). Kants tranzendentale Deduktion und der Möglichkeit von Tranzendentalphilosophie. Frankfurt: Suhrkamp.

Horstmann, R. P. (1984). Die metaphysiche Deduktion in Kants «Kritik der reinen Vernunft». B. Tuschling (ed.), Probleme der «Kritik der reinen Vernunft». Berlín: Walter de Gruyter.

Kant, I. (1996). Critique of Pure Reason. Pluhar, W.S. (trad.), Kitcher, P.W. (ed.). Indianapolis: Hackett.

Kant, I., (1978). Crítica de la razón pura. Ribas, P. (trad.). Madrid: Alfaguara.

Krausser, P. (1981). Kants Theorie der Erfahrung und Erfahrungswissenschaft. Eine rationale Rekonstruktion. Frankfurt/Main: Klostermann.

Kunkelm, E. (1989). Das «Ich denke». Seine gnoselogische Relevanz in Kants «Kritik der reinen Vernuft» und sich hieraus eröffende Perspektiven. Frankfurt: P. Lang.

Röttges, H. (1981). Dialektik als Grund der Kritik. Grundlegung einer Neueinterpretation der Kritik der reinen Vernunft durch den Nachweis der Dialektik von Bedeutung und Gebrauch als Voraussetzung der Analytik, Königstein. Meisemheim am Glan: Hain. 
Glock, H-J. (ed.) (2003). Strawson and Kant. Oxford: Oxford University Press.

Strawson, P. F. (1966). The Bounds of Sense. An Essay on Kant's Critique of Pure Reason. Londres: Methuen.

Theis, R. (1991). Approches de la Critique de la raison pure. Études sur la philosophie théorique de Kant. Hildesheim: Olms.

Tuschling, B. (ed.) (1984). Probleme der «Kritik der reinen Vernunft». Berlín: W. De Gruyter.

Wolff, R. P. (1963). Kant's Theory of Mental Activity. A Commentary on the Transcendental Analytic of the Critique of Pure Reason. Cambridge: Harvard University Press. 


\section{RESUMEN}

En este artículo se pretende aclarar la relación que hay entre la conciencia de sí y la conciencia de objeto en la Crítica de la razón pura de Kant. El problema surge a raíz de la escasa presencia del término autoconciencia (Selbsbewusstsein) en la primera edición de la Crítica. Ello plantea dos posibilidades de interpretación: una que se atiene literalmente al uso kantiano de los términos, y otra que en determinados contextos entiende conciencia también como autoconciencia. Más concretamente, lo que se intenta determinar aquí es si la conciencia de sí requiere de la conciencia de objeto, o si sucede a la inversa. Para ello se realiza un análisis de los conceptos de «unidad sintética», «apercepción trascendental», «conciencia de sí» y «conciencia de objeto», a partir de la segunda edición de la Crítica.

Palabras clave: unidad sintética; apercepción trascendental; conciencia de sí; conciencia de objeto.

\section{ABSTRACT}

This article seeks to clarify the relation between self-consciousness and object consciousness in the Critique of Pure Reason of Kant. The problem arises from the poor presence of the word self-consciousness (Selbsbewusstsein) in the first edition of the Critique. This presents two possible interpretations: one that literally adheres to the Kantian use of the terms, and other that in certain contexts understands consciousness as well as selfconsciousness. More specifically, what we are trying to determine here is whether self-consciousness requires object consciousness, or if the reverse happens. This requires an analysis of the concepts of «synthetic unity», «transcendental appercep- 
tion», «self-consciousness» and «object consciousness» from the second edition of the Critique.

Key words: synthetic unity, transcendental apperception, self-consciousness, object consciousness. 